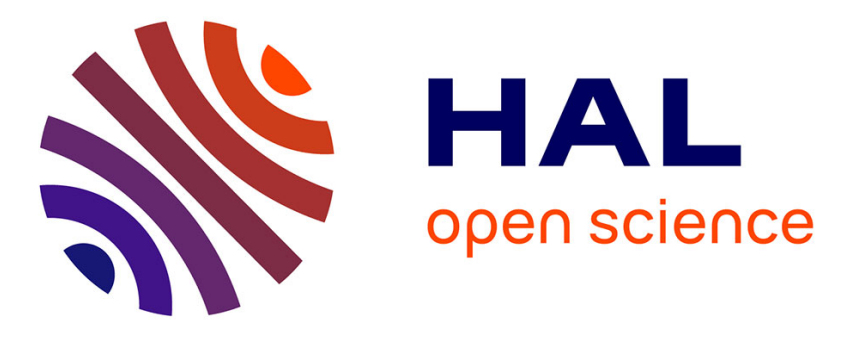

\title{
Predicting playing frequencies for clarinets: a comparison between numerical simulations and simplified analytical formulas
}

Whitney Coyle, Philippe Guillemain, Jean Kergomard, Jean-Pierre Dalmont

\section{- To cite this version:}

Whitney Coyle, Philippe Guillemain, Jean Kergomard, Jean-Pierre Dalmont. Predicting playing frequencies for clarinets: a comparison between numerical simulations and simplified analytical formulas. Journal of the Acoustical Society of America, 2015, 138 (5), pp.2770-2781. 10.1121/1.4932169 . hal01095846v2

\section{HAL Id: hal-01095846 \\ https://hal.science/hal-01095846v2}

Submitted on 2 May 2016

HAL is a multi-disciplinary open access archive for the deposit and dissemination of scientific research documents, whether they are published or not. The documents may come from teaching and research institutions in France or abroad, or from public or private research centers.
L'archive ouverte pluridisciplinaire HAL, est destinée au dépôt et à la diffusion de documents scientifiques de niveau recherche, publiés ou non, émanant des établissements d'enseignement et de recherche français ou étrangers, des laboratoires publics ou privés. 


\title{
Predicting playing frequencies for clarinets: a comparison between numerical simulations and simplified analytical formulas
}

\author{
Whitney L. Coyle \\ Graduate Program in Acoustics \\ The Pennsylvania State University \\ 201 Applied Science Building \\ University Park, PA 16802 \\ Philippe Guillemain and Jean Kergomard \\ Laboratoire de Mécanique et d'Acoustique (LMA, CNRS UPR 7051) \\ 31 Chemin Joseph Aiguier, F-13402 Marseille Cedex 20, France \\ Jean-Pierre Dalmont \\ LUNAM Université, Université du Maine, UMR CNRS 6613 \\ Avenue Olivier Messiaen, 72085 Le Mans Cedex 9, France \\ September 14, 2015
}


When designing a wind instrument such as a clarinet it can be useful to be able to predict the playing frequencies. This paper presents an analytical method to deduce these playing frequencies using the input impedance curve. Specifically there are two control parameters that have a significant influence on the playing frequency, the blowing pressure and reed opening. Four effects are known to alter the playing frequency and are examined separately: the flow rate due to the reed motion, the reed dynamics, the inharmonicity of the resonator, and the temperature gradient within the clarinet. The resulting playing frequencies for the first register of a particular professional level clarinet are found using the analytical formulas presented in this paper. The analytical predictions are then compared to numerically simulated results to validate the prediction accuracy. The main conclusion is that in general the playing frequency decreases above the oscillation threshold because of inharmonicity, then increases above the beating reed regime threshold because of the decrease of the flow rate effect.

PACS numbers: 43.75Ef, 43.75Bc, 43.75Pq 


\section{Introduction}

Musical instruments are more or less sophisticated tools which continue to evolve as technology does. More and more alterations and improvements are being made on these instruments to answer musicians' and manufacturer's wishes concerning sound and playability. Nevertheless, there are still many complicated physical aspects of the instrument that are not yet understood, aspects which could help a musician play "better" or choose a better instrument.

The main objective of the present study is to understand the causes of the difference between playing frequencies and resonance frequencies. Traditional approaches are based upon the assumption that these two frequencies are nearly equal, up to a certain length correction. This correction depends on the note, the excitation parameters and the higher natural modes of the resonator. This paper will provide a choice for a basic (analytical) physical model, from which approximate formulas for the playing frequencies can be derived, and compared to numerical simulation.

In order to modify the playing frequency, the musician can vary multiple control parameters, two of which include the mouth pressure and the action of the lip on the reed. The latter is difficult to introduce directly in a model, but it can be related to the reed-opening size at rest, the reed damping and the reed natural frequencies. Some parameters can be determined experimentally; however in this work, values most often encountered in the literature are used.

This paper studies the first register of the clarinet (first resonance frequency of each tube length) where the playing frequency is mainly determined by the first eigenmode of the pipe. In higher registers, the musician can significantly modify the frequency in order to play "in tune" (the definition of "in tune" is a very intricate issue and is not addressed in this paper). This paper focuses mainly on three causes of discrepancy between playing and resonance frequencies: the flow rate due to the reed compliance, the reed dynamics, and the inharmonicity of the resonator's natural frequencies. A few of these analytical formulas have been known for some time near the oscillation threshold [1]. However, the current work attempts to treat a wider range of playing parameters. The fourth 
The classically cited three equation model is used to solve for three important unknowns: the pressure in the mouthpiece $p_{d}$, the flow rate entering the instrument $u_{b d}$, and the displacement of frequencies might be measured or calculated without temperature gradient and for a temperature different from the effective playing temperature.

This work follows that of two conference papers $[2,3]$. The former treated solely the possibility of gathering the necessary analytical formulas in order to predict a reasonable playing frequency. The latter exploited these analytical formulas in order to create analytical tuning maps. As described, the current work uses a numerical simulation technique to validate the use of these analytical formulas.

The paper begins in Section II by discussing characteristic equations that are widely cited in literature; these equations describe the basic functioning of the clarinet (for example: [17], [1]). This section also provides measured values of the modal parameters of the input impedance of a $\mathrm{Bb}$ clarinet, as inputs for the model. Next, in Section III the playing frequency is studied for the "ideal" case, where it is exactly equal to the resonance frequency, then for the non-ideal case. Analytical formulas for several different frequency corrections are proposed, where the different effects are assumed to be small and therefore can be studied independently. The simulations and comparisons between simulation and analytical formulae are discussed in Sections IV and V respectively. In Section VI the effect of a temperature gradient on the resonance frequencies is briefly studied. In Section VII the total of all four effects is presented. Finally, the paper concludes in Section VIII.

\section{Basic equations}

\section{A The three-equation model for clarinet functioning, with dimensionless variables}

effect, the influence of temperature and temperature gradient, is also discussed as the resonance 
the reed $x_{d}$ (the subscript $d$ refers to variables with dimensions and will be removed when using dimensionless variables in later sections). The first of the three equation model (stated here and explained in further as the section continues:

$$
\begin{aligned}
& u_{b}=\zeta(1+x) \operatorname{sgn}(\gamma-p) \sqrt{|\gamma-p|} \text { if } 1+x \geq 0 \\
& \frac{d x(t)}{d t}=0 \text { if } 1+x \leq 0
\end{aligned}
$$

where $\operatorname{sgn}(x)=|x| / x$. The second,

$$
U_{t o t}(\omega)=Y(\omega) P(\omega)
$$

where,

$$
1 / Y(\omega)=Z(\omega)=\sum_{n} Z_{n}(\omega)=j \omega \sum_{n} \frac{F_{n}}{\omega_{n}^{2}-\omega^{2}+j \omega \omega_{n} / Q_{n}},
$$

where the $\omega_{n}, Q_{n}$ and $F_{n}$ are respectively the resonance frequency, quality factor and "modal factors" obtained from the modal shapes calculated at the input for the $n_{t h}$ impedance peak. And the third,

$$
\frac{1}{\omega_{r}^{2}} \frac{d^{2} x(t)}{d t^{2}}+\frac{q_{r}}{\omega_{r}} \frac{d x(t)}{d t}+x(t)=p(t)-\gamma
$$

where $1 / q_{r}$ is the quality factor and $\omega_{r}$ is the angular frequency of the first reed resonance.

It is assumed that the kinetic energy of the jet entering the instrument is completely dissipated in turbulence during its expansion into the mouthpiece [5]. Then, the acoustic velocity $v$ is related to the pressure difference $p_{m}-p_{d}$, where $p_{m}$ is the mouth pressure, applying the Bernoulli relation: $v=\sqrt{2\left(p_{m}-p_{d}\right) / \rho}$, where $\rho$ is the air density. The height of the reed channel at rest is denoted $H$, assuming the jet cross section to be proportional to $w H$, where $w$ is the effective reed width. The flow rate is therefore: $u_{b d}=w\left(H+x_{d}\right) v$, where the reed displacement $x_{d}$ is 0 at rest and $-H$ when the reed beats. In the static regime, when the pressure difference between the mouth and the 
mouthpiece reaches the closing pressure, the reed beats. This pressure, denoted $p_{M}$, is given by:

$$
p_{M}=H / C_{r},
$$

where $C_{r}$ is the reed compliance (equal to the inverse of the stiffness per unit area).

In order to normalize the pressures, each is divided by the maximum closing pressure $p_{M}$ [4]: $p=p_{d} / p_{M}$ and $\gamma=p_{m} / p_{M}$. The flow rates are normalized by the ratio $p_{M} / Z_{c}$, where $Z_{c}=\rho c / S$ is the characteristic impedance at the input of the tube ( $c$ the speed of sound, and $S$ is the cross section area at the tube input): $u_{b}=u_{b d} Z_{c} / p_{M}$. The reed displacement $x_{d}$ is normalized by $H$ : $x=x_{d} / H$. Therefore $x=-1$ for a reed played in the beating reed regime.

The main dimensionless control parameters are the following: the mouth pressure $\gamma$ and the composite parameter $\zeta$, which is proportional to the maximum flow rate that can enter the tube, and is often seen as the reed opening parameter: $\zeta=Z_{c} w H \sqrt{\frac{2}{\rho p_{M}}}$.

Finally, assuming that the flow is blocked when the reed beats, the dimensionless flow rate $u_{b}$ due to the pressure difference across the reed opening is given by Eq. 1. The second case represents the reed beating against the reed table. According to past studies on clarinet-like instruments, negative flow rate does not occur in the steady-state regime [6, 7]. Equation 1 is the first the 3 equations and represents the so-called "nonlinear characteristic". The remaining equations (Eqs. 3 and 5) are linear and are written in the frequency domain (notated with capital letters). The total flow entering the resonator, denoted $u_{t o t}$, is the sum of the flow rate $u_{b}$ and the the flow rate due to the reed displacement, denoted $u_{r}$. The resonator is described by its input (dimensionless) admittance $Y(\omega)$ as in Eq. 3 and the modal expansion of the dimensionless impedance $Z(\omega)=$ $1 / Y(\omega)$ can be written as $4\left(F_{n}\right.$ has the dimensions of frequency $(\mathrm{Hz})$; for a perfect cylinder of length $\ell, F_{n}$ is equal to $2 c / \ell$, and is independent of the rank of the resonance frequency). From the measured input impedance, the determination of the three coefficients for each mode is explained 
hereafter in Section C. The pressure of each mode $n$ is defined as follows:

$$
P_{n}=Z_{n} U_{t o t}
$$

57 Using, dimensionless quantities, the reed motion is governed by Eq. 5.

58

$$
u_{r d}=-S_{r} d x_{d} / d t
$$

where $S_{r}$ is the reed area contributing to the flow rate (a value which is difficult to determine). In dimensionless quantities, this becomes:

$$
u_{r}=-Z_{c} S_{r} C_{r} \frac{d x}{d t}
$$

This shows that for the non-beating regime, the flow rate due to the reed movement is proportional to the reed compliance $C_{r}$ [1]. The total flow entering the instrument is:

$$
u_{t o t}=u_{b}+u_{r}
$$

At low frequencies, the reed dynamics can be ignored and within the non-beating reed regime conditions:

$$
\frac{d p}{d t}=\frac{d x}{d t}
$$

Considering Eq. (9), the effect of the reed flow is therefore proportional to the acoustic compliance 
of a volume $V_{e q}$ :

$$
u_{r}=-Z_{c} \frac{V_{e q}}{\rho c^{2}} \frac{d p}{d t} .
$$

thus

$$
V_{e q}=\rho c^{2} C_{r} S_{r}
$$

Using Eq. (10), this is equivalent to a compliance in parallel with the input impedance, or to an added air volume at the entry of the instrument, $V_{e q}$. Thus Eq. (3) can be modified as:

$$
U_{b}(\omega)=\left[Y(\omega)+j k \frac{V_{e q}}{S}\right] P(\omega),
$$

with $k=\omega / c$. Reed dynamics could be taken into account by using Eq. (5), but their influence is small, and considering the assumption that the three effects mentioned in the introduction are independent, this correction is ignored. For convenience, the notation $V_{e q}=S \Delta \ell_{e q}$ is often employed: if $\Delta \ell_{e q}$ is smaller than the wavelength, it is in fact an actual length correction at the entrance.

For a reed in the beating reed regime, the reed displacement is limited by the mouthpiece lay, therefore the flow rate is limited as well. When the flow rate is considered, the condition $d x / d t=0$ if $x<-1$ (Eq. 2) replaces the classical condition $u_{b}=0$ if $x<-1$ [4]. Hereafter the work done by Dalmont et al. [8], who published a satisfactory comparison between a simple model and experiment, is summarized. For oscillations in the beating reed regime, the signal of the mouthpiece pressure is not far from a square signal, which is the exact shape for a perfect cylinder when losses, radiation and reed dynamics are ignored. With these assumptions, the pressure takes the following values: $-\gamma$ (when the reed beats), and $+\gamma$ (when the reed is open). For the first harmonic of frequency $\omega_{1}$, the amplitude of the mouthpiece pressure is therefore $2 \gamma / \pi$. The reed displacement is expected to vary between -1 and 0 : thus its first harmonic has an amplitude of 
$1 / \pi$, and $P\left(\omega_{1}\right)=2 \gamma X\left(\omega_{1}\right)$. Therefore:

$$
\frac{d p}{d t} \simeq 2 \gamma \frac{d x}{d t}
$$

Comparing this to Eq. (11) shows that this result is in agreement with the case of non-beating reed regime (before the reed touches the facing): for the simplest theory, a lossless resonator, ignoring reed dynamics, the beating-read threshold (the point, in pressure when the reed first touches the facing) is given by $\gamma=1 / 2$. Finally, both cases of the non-beating and beating reed regime are considered by using the equations:

$$
\begin{gathered}
U_{b}(\omega)=\left[Y(\omega)+j \frac{\omega \Delta \ell_{e q}}{c}\right] P(\omega) ; \\
\text { where } \Delta \ell_{e q}=\frac{\Delta \ell_{0}}{G(\gamma)} \text {, with } \Delta \ell_{0}=\frac{\rho c^{2}}{p_{M}} \frac{S_{r}}{S} H \text { and } \\
G(\gamma)=1 \text { if } \gamma<0.5 ; G(\gamma)=2 \gamma \text { if } \gamma \geq 0.5
\end{gathered}
$$

(see [9]). The function $G$ is here given for an abrupt stop of the reed when closing the reed channel. A more sophisticated function $G$ could be considered in order to take into account a progressive reduction of the moving surface, as observed in practice for high blowing pressures [8].

\section{Extracted modal parameters}

The input impedance has been measured, using a device which was built in Le Mans, France [10]. The source is a piezoelectric buzzer and the pressure in the back cavity of the sound source is measured by a microphone, which gives an estimation of the flow rate. The method to extract the modal parameters from the measured impedance plots is based upon a local optimization procedure (nonlinear least-square algorithm), and is not discussed here. For each mode, the modal frequency 
$f_{n}$, the quality factor $Q_{n}$, and the modal factor $F_{n}$ are extracted. The factor $F_{n}$ is roughly proportional to the fundamental frequency of the notes and is nearly independent of register (the values are almost equal for $n=2$ and $n=1$ ); this behavior is very similar to that of a perfect cylinder. These values are used for both the analytical and numerical calculations throughout this article. $Z_{n}=F_{n} Q_{n} / \omega_{n}$ is the value of the impedance at $\omega=\omega_{n}=2 \pi f_{n}$, when ignoring the effect of the other modes in the series. Moreover, inharmonicity $\eta_{n}$ between Mode $n$ and Mode 1 is defined by the following expression:

$$
\eta_{n}=\frac{\omega_{n}-n \omega_{1}}{n \omega_{1}}
$$

\section{Analytical Formulas for the Playing Frequency}

\section{A Playing frequency in the ideal case}

The ideal case could be considered one where the effects of the reed flow rate and reed dynamics are ignored: $\left(\Delta \ell_{e q}=0, p=x+\gamma\right)$, the linearization of Eq. 1 yields the characteristic equation:

$$
A=Y(\omega) \text { with } A=\frac{\zeta(3 \gamma-1)}{2 \sqrt{\gamma}}
$$

$A$ is the coefficient for the linear term of the nonlinear characteristic $u_{b}(p)$ in Eq. (1), when expanding the function with respect to $p$ around the static regime $p=0$ (if $Z(0)$ is assumed to vanish). Because $A$ is real,

$$
\operatorname{Im}[Y(\omega)]=0,
$$

thus for the first register $\omega=\omega_{1}$, and $A=\operatorname{Re}\left[Y\left(\omega_{1}\right)\right]=A_{t h}$. For small $\operatorname{Re}\left[Y\left(\omega_{1}\right)\right]$, Equation (20) yields the value of the pressure threshold $\gamma_{t h}$ : 


$$
\gamma_{t h} \simeq \frac{1}{3}+\frac{2 R e\left[Y\left(\omega_{1}\right)\right]}{3 \sqrt{3} \zeta}
$$

Above the oscillation threshold, if the resonance frequencies are also harmonically related, the playing frequency $\omega_{p}$ remains the frequency found at the oscillation threshold, i.e. $\omega_{1}$. Also, when the reed dynamics are ignored, there is a static nonlinear characteristic which links the two variables of pressure $p$ and flow rate $u$. Therefore it is possible to use the "reactive power rule" found by Boutillon for bowed instruments [12]:

$$
\sum_{n}\left|P_{n}\right|^{2} n \operatorname{Im}\left[Y\left(n \omega_{p}\right)\right]=0
$$

With Eqs. (1) - (23) from the model, it is now possible to deduce approximations for the difference between natural frequencies and the playing frequencies $f_{p}$ of the clarinet. The frequency difference $\Delta f$ is sought:

$$
\Delta f=f_{p}-f_{1} \text {, with } f_{1}=\omega_{1} /(2 \pi) .
$$

If this value is small enough, the relative difference can be expressed in cents, as follows:

$$
N_{\text {cents }}=1200 \log _{2}\left(f_{p} / f_{1}\right) \simeq \frac{100}{0.06} \frac{\Delta f}{f_{1}} .
$$


This is because one semitone ( 100 cents) corresponds to a ratio of 1.06 . For a cylinder of length $\ell$, a length correction can be defined as:

$$
\frac{\Delta \ell}{\ell}=-\frac{\Delta f}{f_{1}} .
$$

In what follows, the three effects are considered separately, assuming that the frequency shifts (or the length corrections) can simply be added.

\section{Flow rate due to the reed movement}

If both the reed dynamics and the influence of higher order harmonics are ignored, the playing frequency $\omega_{p}$ is given by its value at the oscillation threshold [13]. Because $A$ is real in Eq. (20), Eq. (16) yields a generalization of Eq. (21), as follows:

$$
\operatorname{Im}\left[Y\left(\omega_{p}\right)+j \frac{\omega_{p} \Delta \ell_{e q}}{c}\right]=0
$$

Given that the quality factor of a chosen impedance peak is high enough, only this peak is kept in the modal decomposition around the resonance frequency $\omega_{n}$ and the following approximation is valid [14]:

$$
\operatorname{Im}\left[Y\left(\omega_{p}\right)\right]=\frac{2}{F_{n}}\left(\omega_{p}-\omega_{n}\right) .
$$

Therefore, for the first register, the solution of Eq. 27 is:

$$
\omega_{p}=\frac{\omega_{1}}{1+\frac{\Delta \ell_{e q} F_{1}}{2 c}} .
$$

For a perfect cylinder, if it is assumed that $\omega \Delta \ell / c \approx \tan (\omega \Delta \ell / c)$, the same result is found immediately:

$$
\omega_{p}=\frac{\omega_{1}}{1+\frac{\Delta \ell_{e q}}{\ell}}, \quad \text { with } \omega_{1}=\frac{\pi c}{2 \ell},
$$


if $\omega_{1} \Delta \ell / c \ll 1$. For this particular case, the effect of the flow rate can be viewed as a simple length correction. Eqs. (16) and (29) are those proposed for this first effect, for both a beating reed and a non-beating reed. Finally, it is written:

$$
\text { Ncents }_{\text {flow }}=-\frac{100}{0.06} \frac{F_{1} \Delta \ell_{0}}{2 G(\gamma) c}
$$

\section{Reed dynamics}

When reed dynamics are considered $A \neq Y\left(\omega_{p}\right)$ at the threshold of oscillation. The study of the influence of the reed dynamics on the oscillation threshold (frequency and mouth pressure) has been done by Wilson and Beavers [15], and extended by Silva et al. [16] by adding the effect of the reed flow rate. The results are valid for the case of strong reeds (e.g., organ reeds, with small reed damping), and weak reeds (e.g., woodwind reeds, with high damping by the lips). This method involves the linearization of Eq. (1), and solving the characteristic equation. Here the case of high damping, i.e., large $q_{r}$ is considered. For a cylinder, the result at the oscillation threshold was given in the form of a length correction in [16, Eq. (31)]: $\Delta \ell=q_{r} \zeta /\left(k_{r} \sqrt{3}\right)$, where $k_{r}=\omega_{r} / c$. The method of Ref. [16] leads to the following result if the tube is a one-mode oscillator:

$$
\omega_{p}=\omega_{1}\left[1-\frac{\zeta F_{1}}{2 \sqrt{3}} \frac{q_{r}}{\omega_{r}}\right] .
$$

Notice that Nederveen tried to describe this effect, but he considered a reed with high damping and an infinite natural frequency [1]. In his eq. (25.23), the length correction is denoted $\Delta \ell_{\tau}$, and increases with the reed opening at rest, which is in accordance with the above result in Eq. 32. Further comparison with his result is difficult because a different nonlinear characteristic was used.

Equation (32) is valid at the threshold of oscillation, for a non-beating reed and a very small excitation pressure $\gamma$. Nevertheless, Kergomard and Gilbert [18], using the harmonic balance method 
analytically (limited to the first harmonic), found the following dependence on the excitation pressure:

$$
\omega_{p}=\omega_{1}\left[1-\frac{\zeta F_{1}}{2 \sqrt{3}} \frac{q_{r}}{\omega_{r}}\left[1+\frac{3}{4}\left(\gamma-\gamma_{t h}\right)\right]\right] .
$$

This is the beginning of a series expansion above the oscillation threshold $\gamma_{t h}$, consequently the formula is not necessarily valid at high values of $\gamma$, i.e. Eq. 33 is obtained for the non-beating reed regime only. When losses in the pipe are ignored, the value of the oscillation threshold $\gamma_{t h}$ is given by Silva et al. [16]. However, the current work uses small perturbation reasoning and therefore an approximate version of Silva's threshold of oscillation can be used, as in Eq. (22).

This effect can be transformed into a simple change in frequency, in cents, as well and is represented as follows:

$$
\text { Ncents }_{\text {dynamics }}=-\frac{100}{0.06} \cdot \frac{\zeta F_{1}}{2 \sqrt{3}} \frac{q_{r}}{\omega_{r}}\left[1+\frac{3}{4}\left(\gamma-\gamma_{t h}\right)\right] .
$$

\section{Effect of the inharmonicity of the resonator}

If the resonance frequencies are not exactly harmonic, the playing frequency changes with the level of excitation [19].

The method is valid for any given shape of the nonlinear characteristic $u=F(p)$, for both nonbeating and beating reed regimes. In this case, because reed dynamics are ignored, this (static) characteristic exists $(x=p-\gamma$ in Eq. (5)). For clarinet-like instruments with weak inharmonicity (small $\eta_{n}$ ), the summation in Eq. (23) can be limited to odd harmonics. It is possible to use Eq. (28) near every resonance frequency, seeking the playing frequency in the form:

$$
\omega_{p}=\omega_{1}(1+\varepsilon)
$$


At the first order in $\eta_{n}$ and $\varepsilon$, Eq. (23) yields:

$$
\sum_{n \text { odd }} n^{2}\left|P_{n}\right|^{2} \frac{\varepsilon-\eta_{n}}{F_{n}}=0
$$

For cylindrical instruments, because the modal factor $F_{n}$ is nearly independent of $n$ (and equal to $2 c / \ell$ ) and because $\eta_{1}=0$, the final result for the playing frequency is Equation (35), with:

$$
\varepsilon=\frac{\sum_{n \text { odd } \geq 3} \eta_{n} d_{n}}{1+\sum_{n \text { odd } \geq 3} d_{n}} \text { with } d_{n}=n^{2}\left|\frac{P_{n}}{P_{1}}\right|^{2} .
$$

(note that for a square signal $d_{n}=1$ for every odd $n$ ). If the dependence of the spectrum with respect to the excitation pressure is known, it is possible to deduce the variation in the playing frequency. Approximate formulas for clarinet-like instruments were given by Kergomard et al [20]. The decrease of the higher harmonics is always faster than in the case of the square signal, therefore it is reasonable to search for an approximate formula by limiting the series to the third harmonic only. The ratio of the amplitude $P_{3} / P_{1}$ is given by Eq. (21b) of [20]:

$$
\frac{P_{3}}{P_{1}}=-\frac{1}{3} \frac{1}{1+z}
$$

where,

$$
z=\frac{Y_{3}-Y_{1}}{A-Y_{1}} \text { and } Y_{n}=\frac{1}{Z_{n}}
$$

Experimentally, there have been measurements of $P_{3} / P_{1}$ smaller than $1 / 3$, but this formula is a good approximation for both the non-beating and beating reed regimes. Finally, a first order approximation is found:

$$
\varepsilon=\frac{\eta_{3}}{1+|1+z|^{2}}
$$

At the threshold $A=Y_{1}, \varepsilon=0$ (the signal is sinusoidal), and $Y_{1}$ is real. For large excitation pres- 
sure, $z$ tends to zero, and $\varepsilon$ to $\eta_{3} / 2$. Notice that because the reasoning is based upon a perturbation at the first order, the values of $Y_{3}$ and $Y_{1}$ can be determined without inharmonicity, i.e. they are real.

Refinements to this formula would be quite intricate (as an example the formula for the 5th harmonic is very complicated, see [20, Eq. (21b)]). Nevertheless, this formula exhibits the sense of variation of the effect on inharmonicity of the second peak and doesn't necessarily warrant calculation beyond this. As before it is helpful to transform the frequency changes into a simpler value, in cents:

$$
\text { Ncents } s_{\text {inharmonicity }}=\frac{100}{0.06} \cdot \frac{\eta_{3}}{1+|1+z|^{2}}
$$

\section{Numerical Simulations}

In order to validate the results of the analytical models, a synthesis model which is real-time compatible is chosen and based on the one proposed by Guillemain et al.[11]. It is based on the physical model presented in Section II. This model provides a straightforward digital transposition of each part of the physical model. Many simplifications are made regarding the functioning of the reed and all parameters explicitly stated in this article are used when calculating the synthesized impedance peaks. The three equations $(1,5,16)$ are solved for the three dimensionless quantities $u_{b}, p$, and $x$, defined in Section II.

Eqs. (1) and (5) are discretized according to [11], which leads to an explicit computation scheme. In what follows, we calculate two quantities, denoted $J$ and $V$, which only depend on the past values of the variables, and consequently are known at time sample $m$.

The quantity $\mathbf{J}$ is defined as follows:

$$
J=b_{1_{a}} p_{e}(m-1)+a_{1_{a}} x(m-1)+a_{2_{a}} x(m-2)
$$


where the coefficients $b_{1_{a}}, a_{1_{a}}$ and $a_{2_{a}}$ are defined analytically as functions of the resonance frequency $\omega_{r}$ and quality factor $q_{r}$ of the reed and $f_{e}$ is the sampling frequency:

$$
a_{0_{a}}=\frac{f_{e}^{2}}{\omega_{r}^{2}}+\frac{f_{e} q_{r}}{2 \omega_{r}}, \quad b_{1_{a}}=\frac{1}{a_{0_{a}}}, \quad a_{1_{a}}=\frac{\frac{2 f_{e}^{2}}{\omega_{r}^{2}}-1}{a_{0_{a}}}, \quad a_{2_{a}}=\frac{\frac{f_{e} q_{r}}{2 \omega_{r}}-\frac{f_{e}^{2}}{\omega_{r}^{2}}}{a_{0_{a}}} .
$$

Concerning the resonator, at each time sample $m$, each impedance mode $n$ (Eq. (4)) is discretized according to the invariance of the impulse response method after an estimation of the modal parameters $F_{n}, \omega_{n}$ and $Q_{n}$. This is done with the coefficients $b_{n 0}, b_{n 1}, a_{n 1}$, and $a_{n 2}$ computed analytically as functions of the modal parameters (see Eq.(4)). Denoting $e_{a}=\exp \left(-\omega_{n} /\left(2 Q_{n} f_{e}\right)\right)$ and $c_{w}=\cos \left(\omega_{n} / f_{e}\right)$, they are:

$$
b_{n 0}=F_{n} / f_{e}, \quad b_{n 1}=-F_{n} / f_{e} e_{a} c_{w}, \quad a_{n 1}=2 e_{a} c_{w}, \quad a_{n 2}=-e_{a}^{2},
$$

and Eq. (3) is expressed as follows:

$$
\begin{aligned}
p_{n}(m) & =b_{n 0} u_{t o t}(m)+V_{n} \\
\text { with } V_{n} & =b_{n 1} u_{t o t}(m-1)+a_{n 1} p_{n}(m-1)+a_{n 2} p_{n}(m-2) .
\end{aligned}
$$

Then

$$
\begin{aligned}
p(m) & =\sum_{n=1}^{N} p_{n}(m)=b_{M 0} u_{t o t}(m)+V \\
\text { where } b_{M 0} & =\sum_{n=1}^{N} b_{n 0} \text { and } V=\sum_{n=1}^{N} V_{n} .
\end{aligned}
$$

Previous work [11] did not include the reed flow effect. This was simpler for the non-beating reed regime but a more difficult task for the beating reed regime. In order to describe the beating reed regime, a point where the velocity is zero (for a given pressure) is created, at the time where 
the reed hits the table for the first time. The two sources of flow are then added together, $u_{t o t}(m)=$ $u_{b}(m)+u_{r}(m)$. In the discrete time domain, Eqs. (2) and (9) are written as:

$$
\text { if } \begin{aligned}
1+x(m) & \leq 0 \text { then } x(m)=x(m-1) . \\
u_{r}(m) & =-\lambda[x(m)-x(m-1)],
\end{aligned}
$$

where $\lambda=-f_{e} \Delta \ell_{0} / c=-f_{e} Z_{c} S_{r} C_{r}$ is a dimensionless coefficient. For the simulations $\lambda=-0.7$ has been chosen: this value corresponds to $\Delta \ell_{0}=5.5 \mathrm{~mm}$, as explained in the next section.

Here, unlike in the analytical formulas, the reed dynamics model is valid above the beating reed regime due to added stipulations within the simulations, one being the inclusion of the ability for the reed to touch the table of the mouthpiece and at that point have a velocity of zero, as well as the ability to have negative flow.

As a second step, the reed displacement discretization scheme is [11]:

$$
\begin{aligned}
& x(m)= J ; \\
& \text { if } 1+x(m)<0, x(m)=x(m-1) ; \\
& u_{r}(m)=\lambda(x(m)-x(m-1)) ; \\
& W=\zeta(1+x(m)) ; \\
& p=b_{M 0}\left(u_{b}+u_{r}\right)+V ; \\
& u_{b}=\operatorname{Wggn}(\gamma-p) \sqrt{|\gamma-p|} .
\end{aligned}
$$

Here for the sake of clarity the time sample $m$ has been omitted in the variables $p, u_{b}$, and $u_{r}$. As a third step, these equations are then transformed into two second order polynomial equations in $u_{b}$ corresponding to either positive or negative values of $u_{b}$, yielding the final solution: 


$$
\begin{aligned}
& u_{t o t}=\frac{\operatorname{sgn}\left(\gamma-V-b_{M 0} u_{r}\right)}{2}\left(-b_{M 0} W^{2}+\right. \\
& \left.W \sqrt{\left(b_{M 0} W\right)^{2}+4\left|\gamma-V-b_{M 0} u_{r}\right|}\right)+u_{r} ; \\
& p_{n}=b_{n 0} u_{t o t}+V_{n} ; \\
& p=\sum_{n=1}^{N} p_{n} .
\end{aligned}
$$

\section{Results: comparison between analytical formulas and simu-}

\section{lation}

The terminology used in this section refers to notes of the first register of the Bb soprano clarinet with the numbers $1-19$. Note 1 represents the lowest note on the $\mathrm{B} b$ clarinet, the fingered $\mathrm{E}$ that produces a frequency near $146 \mathrm{~Hz}$ (considering the equal-tempered scale) and Note 19 represents the highest note in the first register, the fingered $\mathrm{B} b, 415 \mathrm{~Hz}$.

The following values are used for each note: vibrating surface area of the reed, $S_{r}=6.5 \cdot 10^{-5}$ $m^{2}$. The quality factor of the reed is $Q_{r}=5$ (the inverse being $q_{r}=0.2$ ), the resonance frequency of the reed is $f_{r}=2400 \mathrm{~Hz}$, and $\zeta=0.3$; they are reasonable choices for the playing parameters of the reed and the environment based on previous work by Wilson and Beavers [15] and Dalmont [21]. Most of these parameters are difficult to measure in an experimental setting so it is best to choose well accepted values at this time. Specifically concerning the effect of the reed movement, it is difficult to estimate it from the knowledge of the reed area contributing to the flow rate, $S_{r}$. In this paper, an empirical value for the parameter $\Delta \ell_{0}$ of $5.5 \mathrm{~mm}$ is chosen: this allows to find a total length correction around $7 \mathrm{~mm}$ (see discussion below), in accordance with experimental results obtained by Dalmont et al. [21]. Each of these formulas discussed in the previous sections leads to a specific frequency correction that can be added together to become the calculated playing 
frequency of the clarinet.

In order to compare to the numerical and analytical results for each effect it was necessary to find a way to suppress individual effects within the simulations. To suppress the reed induced flow effect within the simulations it is sufficient to say that $\lambda$ is zero. To suppress the reed dynamics a large value for $\omega_{r}$ and a low value for $q_{r}$ may be chosen. This should be done carefully since an extreme choice for these two values could force the clarinet to play in a different register. To suppress the effect of inharmonicity the frequency of the second peak of the impedance spectrum is set to be three times that of the first peak.

Figures 1, 2 and 3 represent the frequency correction, in cents, for three different notes in the first register of the $\mathrm{B} b$ clarinet: notes 1, 12 and 17, respectively. The figures compare the calculated playing frequency (either from the analytical formulas or the simulations) to the extracted modal resonance frequencies (the difference between these and the equal-tempered scale for a chosen temperature is shown later in Figure 6) for each particular choice of parameters. Notice that the frequency corrections are listed separately (reed induced flow, reed dynamics and inharmonicity) as well as totaled. Recall that, although the figures give a maximum value of $\gamma=1$, the analytical reed dynamics Eq. (33) is only accurate until $\gamma \approx 0.5$ (this value corresponds to the simplest expression of the beating reed threshold). Each note of the clarinet exhibited a negative inharmonicity $\left(\eta_{3}<0\right)$ though the two notes, 12 and 17 were chosen since they offer respectively the maximum and minimum values of inharmonicities possible for the first register of the instrument in question. Note 1 was chosen since it can exhibit the total resonator effects with no open tone holes.

Each figure shows the expected trends for each effect: the value for inharmonicty effect is zero at the threshold of oscillation (the lower limit in $\gamma$ ) and increases with increasing blowing pressure (more-so for lower notes), the reed induced flow has the greatest effect on the playing frequency and the effect of the reed dynamics is generally quite small and does not depend heavily on the value of the blowing pressure, $\gamma$. Further, the two latter effects increase with the fundamental frequency of the note, but at low frequencies and low excitation level, these effects seem to be 
independent of frequency and excitation level and can be considered an actual length correction (as in the case when the clarinet is tuned by lengthening at the joints).

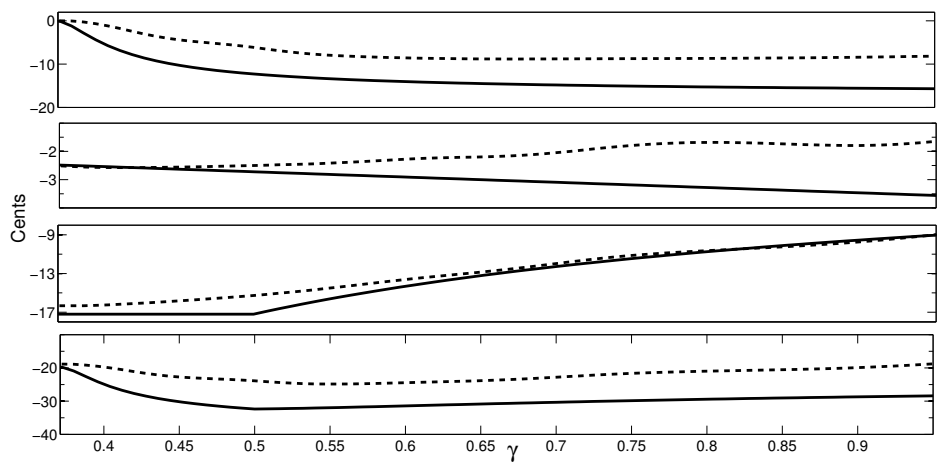

Figure 1: The frequency difference $N_{\text {cents }}$ between the 1st impedance peak frequency (resonance frequency) and the playing frequency for Note 1 (fingering for $\mathrm{E}$ ) of the clarinet. Note 1 values: $\zeta=0.3, \eta_{3}=-0.0201, F_{1}=1243 \mathrm{~Hz}$ and $f_{1}=146 \mathrm{~Hz}$. Solid lines represent the analytical results, while dotted lines represent the numerical results. There are four plots to represent (beginning at the top of the figure): The inharmonicity effect, the reed dynamics effect, the reed induced flow effect. The bottom plot is the total of all three effects. The x-axis is the same for all four plots and is an increasing blowing pressure $\gamma$. The y-axis is different for each plot, ranging anywhere from 0 to -40 cents different.

For the numerical simulation, a question arises concerning the number of modes taken into account. In our simulation, only two significant modes were used in the numerical simulations. This can affect the results especially for the lower notes where there are a large number of significant modes present, perhaps up to seven. Conversely, this is not as much of a problem for higher notes since there are perhaps only two or three significant modes present in the impedance spectrum. Convergence tests were run in order to verify that the use of two modes would produce a sufficient amount of accuracy. For Note 1, a perfect convergence is found when the number of modes is successively chosen to be 2,4 , and 6 , and the result with 2 modes seems satisfactory for the effects of both reed flow and reed dynamics. Concerning the inharmonicity effect, the result with 6 modes is closer (by 2 cents) to the analytical result than that with 2 modes. However this is not significant, because the addition of the 3rd mode (with frequency 5 times higher than the fundamental) in the 


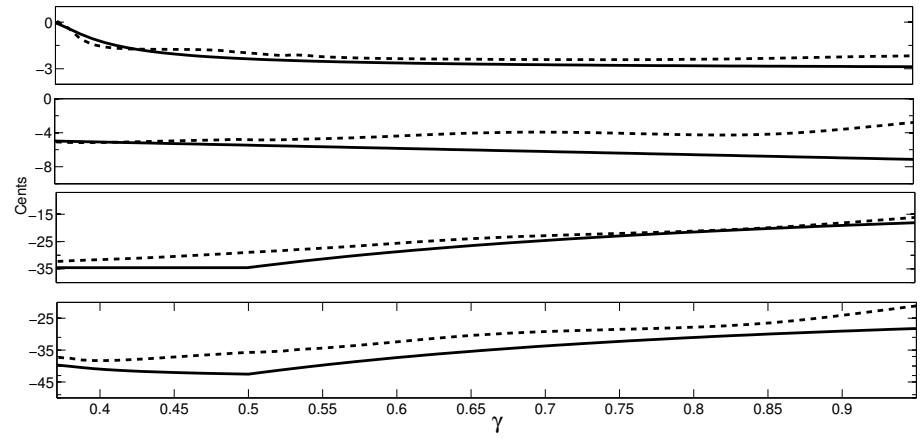

Figure 2: The frequency difference $N_{\text {cents }}$ for Note 12 (fingering for Eb) of the clarinet. Note 12 values: $\zeta=0.3, \eta_{3}=-0.0036, F_{1}=2490 \mathrm{~Hz}, f_{1}=277 \mathrm{~Hz}$. The different subfigures match that of Figure 1.

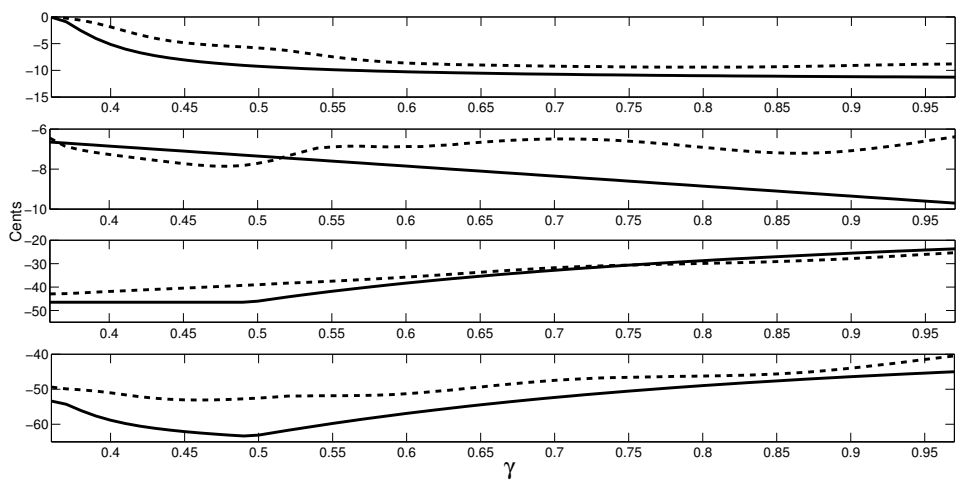

Figure 3: The frequency difference $N_{\text {cents }}$ for Note 17 (fingering for $\mathrm{A} b$ ) of the clarinet. Note 17 values: $\zeta=0.3, \eta_{3}=-0.0142, F_{1}=3338 \mathrm{~Hz}, f_{1}=369 \mathrm{~Hz}$. The different subfigures match that of Figure 1. 
analytical calculation for Note 1, following the work in [20], did not enhance accuracy. Actually the inharmonicity of the 3rd mode is negative $\left(\eta_{5}=-0.052\right)$ and the discrepancy between analytical and numerical result would increase when this mode is taken into account. In addition, an analytical approximation for the amplitude of the 5th harmonic is quite complicated to derive, and is not included in this work.

Another difficulty can arise from the choice of the reed (first) resonance frequency and has been found to be critical for higher notes: if this frequency is too low (or $q_{r}$ is too small), the numerical model may sometimes produce oscillations in another register.

Comparing the results from the analytical formulas and the simulations, the hope is that these results for each effect, as well as the total frequency difference will be as similar as possible in shape and value. Realizing that the actual value in cents of a "Just Noticeable Difference" (JND) $[22,34]$ in pitch varies depending on context and content of sounds being played, for this study, the benchmark used is that the difference between analytical and numerical simulation curves be less than 10 cents.

A few general comments can be made for the discrepancy between analytical and numerical results, for each of the three effects:

- For the inharmonicity effect, the general tendency is satisfactory, especially for higher notes. For Note 1 (see Fig. 1), the order of magnitude of the discrepancy is 10 cents (see above), but the variation with the excitation pressure $\gamma$ is well predicted. The discrepancy here for lower notes is found to be the most important of the present study, and explains the lower quality of the results for the total of the three effects for Note 1 . This is probably due to the limitation to two harmonics in the analytical formula. Notice though, that [17, p. 441], showed that if the most basic approximation for this effect is considered, the order of magnitude should be near $\varepsilon=\eta / 2$, which is indeed the case for the three notes studied.

- For the reed flow, the hypothesis leading to the analytical formula for the beating reed regime 
[8] is validated, because the discrepancy is limited to 2 or 3 cents. The decrease of the length correction with increasing $\gamma$ is well predicted. However the agreement between analytical and numerical results is less satisfactory for the note 17 .

- For the reed dynamics, the effect is rather small as is the difference between analytical and numerical simulation. The slight frequency decrease just above the threshold is correctly predicted [18]. It is more surprising that, for the lower notes at least, the discrepancy remains small for a beating reed since no analytical formula was derived for this case (the formula for the non-beating reed regime is simply continued).

Overall, for all notes in the first register of the clarinet, the validation of the analytical formula by the simulation is satisfactory. The two main delicate points remain in the inharmonicity effect for the lower notes and the reed dynamics effect for the higher notes.

In Ref. [23] Nederveen and Dalmont compared experiment with a numerical computation for 3 fingerings of a clarinet. The 3 fingerings give the same note, but inharmonicity was different (between -0.04 and -0.007). Their model is similar to that of the present paper, but a comparison between the two models and the solving methods is out of the scope of the paper. However, using some data used in their computation, the main trends of the analytical results are in qualitative agreement with the computation of Ref. [23]. In particular, we have found that the effect of the reed dynamics is weak, except for fingering of the top of the first register. For fingering 17, between the oscillation and the beating-reed thresholds, both the reed dynamics and inharmonicity contributes, with the same order of magnitude to the decrease of the playing frequency. As observed in [23], the inertia of the reed increases with frequency, and it is likely that its influence is even more important on the second register, leading to an increase of the length correction along the second register. In general, the playing frequency decrease above the oscillation threshold is mainly due to inharmonicity, while the increase above the beating reed threshold is due to the decrease of the flow rate effect. For high mouth pressures, the predicted frequency increase is exaggerated by 
where $\delta \rho$ is the variation of density and $\ell$ is the equivalent length of the instrument at the particular impedance peaks above the second one in Eq. (40), and to the interaction between the three effects.

\section{The temperature gradient effect}

Until now, the calculations for playing frequencies are based on the resonance frequencies measured at $20^{\circ} \mathrm{C}$, for both numerical and analytical calculations. It is intuitive that the air closest to the mouthpiece, having just left the air-column of the instrumentalist, would have a higher temperature than the air that will exit the instrument for the lowest note on the clarinet. In general, an average temperature over the instrument was used in predictions of playing frequencies since it was considered to not vary greatly with a change in note [24]. Previous research stated that the temperature could be seen as an average over the instrument since it does not vary greatly with a change in note [25, 26]. However, recent measurements by Noreland [27] show that these temperature differences can be as much as a $9^{\circ}$ Celsius difference from the top of the barrel $\left(T_{0}=\right.$ $\left.31^{\circ} \mathrm{C}\right)$ to the bottom of the bell $\left(22^{\circ} \mathrm{C}\right)$, as well as that the temperature profile is linear and nearly independent of note (i.e. the number of open holes). Thus the consideration of this effect is simple.

In order to take the temperature value and gradient into account, the frequency shift due to a temperature change of $11^{\circ} \mathrm{C}$ (from $22^{\circ} \mathrm{C}$ to $31^{\circ} \mathrm{C}$ ), is first computed, then the effect of the temperature decrease inside the instrument. Therefore first a correction to the final results is simply added which is equivalent to the effect of a $\Delta T=11^{\circ} \mathrm{C}$ change in temperature, nearly 33 cents.

The calculation for the effect of the gradient is similar to that for flute-like instruments done by Coltman [28], replacing the cosine function by the sine function:

$$
\Delta \ell=\int_{0}^{\ell} \frac{\delta \rho}{\rho} \sin ^{2}(k x) d x
$$

the analytical formulas. The reason for this is probably due to the insufficient consideration of the 
note. Using the measured linear temperature gradient from [27]:

$$
T(x)=T_{0}\left[1-\kappa \frac{x}{L}\right],
$$

where $L$ is the total length of the instrument. $\kappa$ is a constant and $x$ is the distance from the top of the barrel a useful analytical formula is found (from Eq. 57)). The equivalent length correction for the temperature gradient inside the clarinet is found to be:

$$
\frac{\Delta \ell}{\ell}=\left(\frac{1}{4}+\frac{1}{\pi^{2}}\right) \kappa \frac{\ell}{L}
$$

where $\kappa=9 /\left(T_{0}+273\right)$ (based on measurements in [27]). In this calculation, the formula $k \ell=\frac{\pi}{2}$ is assumed. The specific effect of the $\mathrm{CO}_{2}$ content and the percent humidity are not included in these calculations since they are small values and can be assumed to have a very small effect $[29,28,30,31]$. More specifically, Fuks states that the effect of gas changes can cause as much as a 20 cent decrease in fundamental frequency [30]. However, his study focused on a long sustained note whereas this work is interested in a small portion of the playing frequency in the steady-state regime and not on the evolution of pitch throughout a sustained note.

The final result, in cents, for this effect is:

$$
\begin{aligned}
& \text { Ncents } \text { temperature }= \\
& \frac{100}{0.06}\left(\left(\frac{1}{4}+\frac{1}{\pi^{2}}\right) \kappa \frac{\ell}{L}-\frac{1}{2} \frac{\Delta T}{\left(T_{0}+273\right)}\right) .
\end{aligned}
$$

\section{Total of four effects}

We now consider the four effects on the first register of a clarinet whose impedance curves have been measured for each fingerings.

Figure 4 and 5 show the total length corrections, in $\mathrm{mm}$, as a function of note in the first 


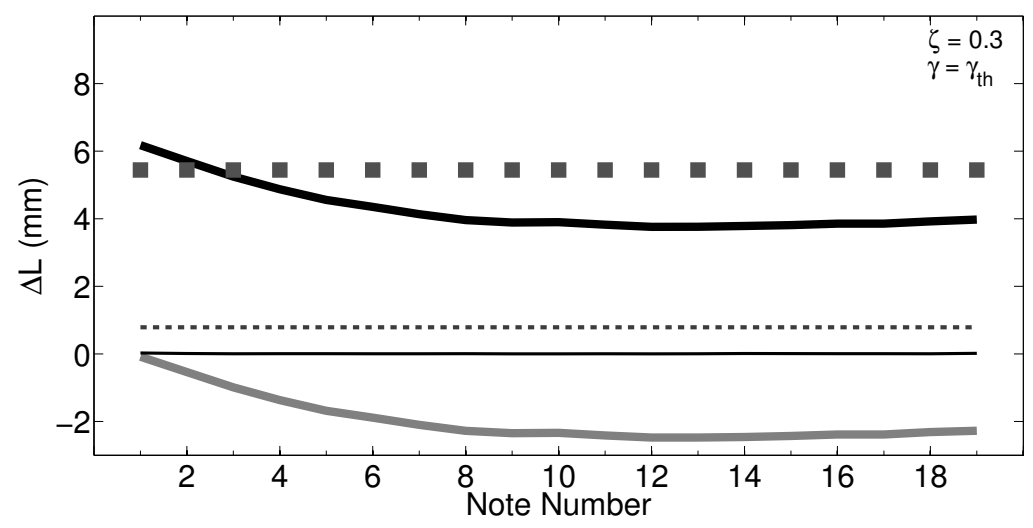

Figure 4: Analytical length corrections $(\Delta \ell)$ values, in $\mathrm{mm}$, as a function of note number, in the first register, at the threshold of oscillation. Black thin line: inharmonicity effect, Thin dashed line: reed dynamics, Dark grey solid line: temperature effect, Grey squares: reed flow effect and finally, the thick black solid line is the total of all four effects. Realize that the plot for the inharmonicity effect is at zero for the $\gamma=\gamma_{t h}$ (a value that changes depending on Note number, generally around $0.33)$.

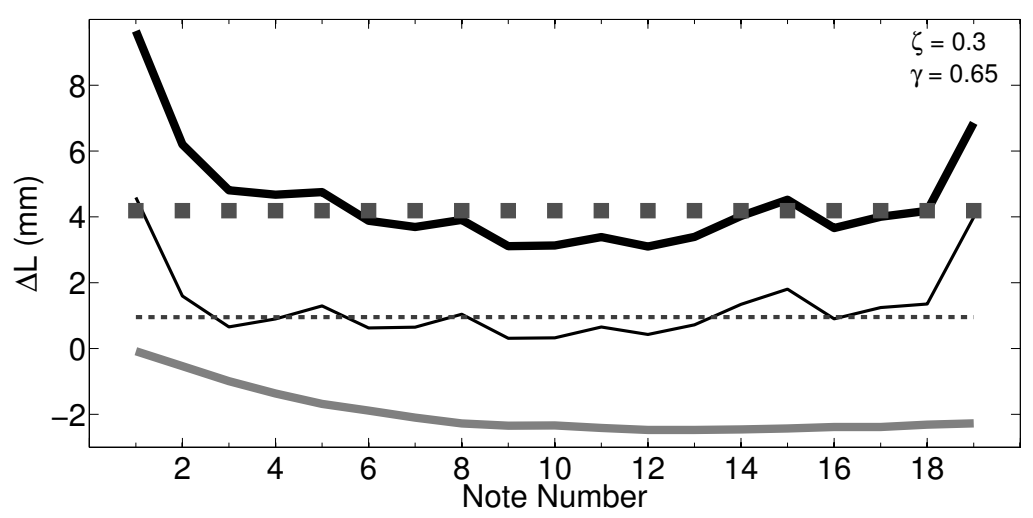

Figure 5: Analytical length corrections $(\Delta \ell)$ values, in $\mathrm{mm}$, as a function of note number, in the first register. The color / line scheme matches that of Figure 4. The data are represented for $\gamma=0.65$. 
register of the clarinet for two different values of $\gamma$. This total length correction is calculated by the following formula:

$$
\Delta \ell_{\text {total }}=-\frac{\Delta f}{f} \ell, \text { where } \ell=\frac{2 c}{F_{1}}
$$

The value for $\zeta$ remained 0.3 and the value for $\gamma$ was chosen to be either $\gamma_{t h}$, the threshold of oscillation for each given note, or $\gamma=0.65$. In Figure 4, notice that for $\gamma=\gamma_{t h}$ the curve for the inharmonicity remains at zero, this is because the inharmonicity of the resonator has no effect at the threshold of oscillation. For the reed induced flow effect, as expected, a value near $5.5 \mathrm{~mm}$ is found. As required, the total magnitude of the length corrections for these notes corresponds well to the work done by Dalmont et al in [21] which showed length corrections totaling $7 \mathrm{~mm}$ to $10 \mathrm{~mm}$ (since this work was based on the use of an artificial mouth it does not consider the temperature effect which is discussed in section VI). The effect of inharmonicity is similar for each note creating a difference between analytical predictions and numerical simulations no larger than 15 cents for the total range of $\gamma$.

These figures show that the formulas that represent the reed induced flow effect is stable and offers the largest frequency shift of the three effects. This was to be expected based on the work in [8] and also shows the change in behavior above the beating reed regime. For the case of reed dynamics, not surprisingly, the effect is small for every note and nearly linear as a function of $\gamma$. It is obvious from these figures that the effects are very much dependent on note (length of resonator). This is intuitive for the clarinetists since as the effective length of the instrument is shortened, there are more factors influencing the playing frequency and therefore more compensation needed in order to play "in-tune". Figure 6 shows the same total effects as in previous figures but represents the frequency shift vs. the equal-tempered scale. For comparison, the top line in Figure 6 also offers the first extracted modal frequencies for each note. 


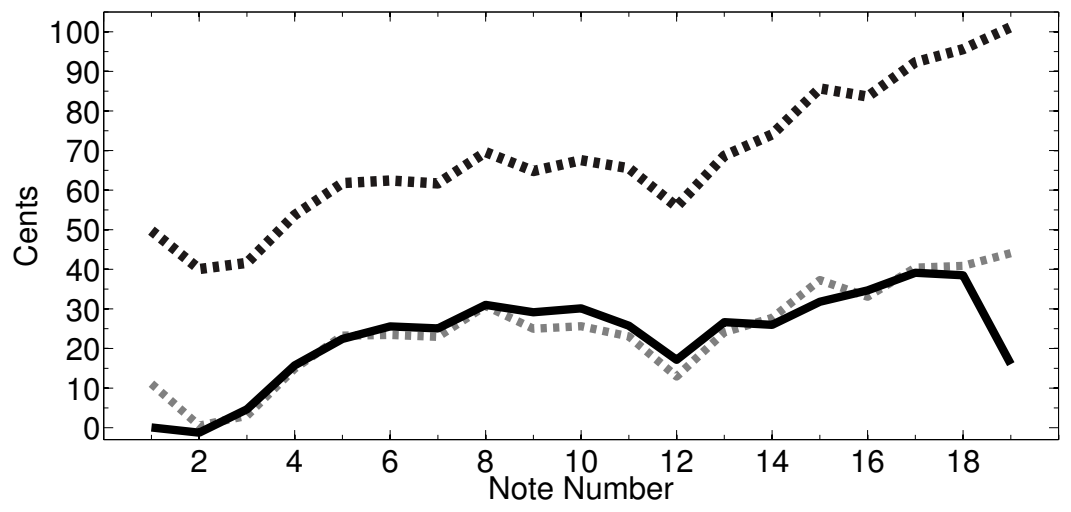

Figure 6: Top line (black dotted) is the difference, in cents between the first resonance frequencies and the equal-tempered scale frequencies using an averaged temperature of 26.5 degrees Celsius. The lower two lines represent the total frequency shift vs. the equal-tempered scale, in cents, as a function of note number, for the first register, where $\gamma=0.65$ (black solid line) and $\gamma_{t h}$ (grey dotted line).

\section{Conclusions}

The analytical formulas presented here are computationally fast, accurate and can be used in a number of situations in order to study the playing frequency of the clarinet.

An important point to highlight is that the computation time for the full range of $\gamma$ and $\zeta$ using the numerical simulations is about three hours for all notes in the first register. Using the analytical formulas for the full range of control parameters can complete the task in less than two minutes. This is an extremely valuable decrease in computing time and makes this a valid and useful method for predicting clarinet playing frequencies. However, difficulties can occur because of the estimation uncertainty of the reed resonance frequency and the inharmonicity effect for the lowest notes.

The difference in predictions of the numerical simulations and analytical formulas are small and the results presented here are sufficient enough to warrant the sole use of the analytical formulas to predict playing frequencies. There are, nevertheless, certain aspects that were not taken into account in this work, including the nonlinearity of toneholes and the effect of the vocal tract on 
the playing frequency. The influence of the nonlinearity of the holes on the playing frequency can probably be ignored because the particular clarinet studied (professional level) employs undercut toneholes in order to avoid this effect [24]. Further, it is reasonable to ignore the effect of the vocal tract, which has been shown to have little effect on the lower register of the clarinet [32]. Finally, a limitation of this work comes when ignoring the effect of $\mathrm{CO}_{2}$ and humidity and that the effect of temperature gradient is based only on the work by Noreland [27]. Taking detailed measurements which include not only the temperature profile but the $\mathrm{CO}_{2}$ and humidity content as well could make the playing frequency predictions more accurate.

An important limitation is probably in the evaluation of the reed flow effect and its evolution with the mouth pressure (i.e., the empirical function $G$ in the model). Indeed this is a control parameter that could be used by the player to adjust the pitch of the note (but is not ideal for the majority of musical contexts). Furthermore, the fact that the mouthpiece table facing angle and actual dimensions of the vibrating surface of the reed are largely estimated adds to the uncertainty in the final results. Another important limitation is the influence of higher order resonance peaks which may sometimes influence the pitch in a complicated manner. This is a subject for further research. In order to use the analytical formulas (and simulations), a difficult task remains in determining the main parameters (reed characteristics, etc.). Some of these could be deduced by using an artificial mouth, assuming a perfect cylinder. Obviously this task is even more difficult when attempting to measure with an instrumentalist. The important point is that these results can help to discern the inhomogeneity of tuning from the inhomogeneity of the modal parameters. Concerning this topic, the goal of this work joins that of the paper by Almeida et al [33], who used an experimental approach to show playing frequencies based on different input values. Comparing these results to measurements of the playing frequencies using an artificial mouth apparatus as well as from actual musician playing tests could validate the practical applications of the analytical formulas in the clarinet manufacturing process. 


\section{Acknowledgments}

The authors would like to thank the Pennsylvania State University Graduate Program in Acoustics and Dr. Daniel Russell, funding from the NSF-GRFP Grant No. DGE1255832 and the NSFGROW, The Chateaubriand Fellowship and The ARCS Foundation, and finally the French ANRCagima project and all of its members.

\section{References}

[1] C. J. Nederveen. Acoustical aspects of woodwind instruments. 160 pages. N. Illinois Univ. Press, DeKalb, Illinois, 1998.

[2] W. Coyle, P. Guillemain, and J. Kergomard. Rapid creation of tuning maps for a clarinet using analytic formulas,. Proceedings of the International Symposium on Musical Acoustics, 295-299, 2014.

[3] W. Coyle, J. Kergomard, P. Guillemain, C. Vergez, and A. Guilloteau. An attempt at predicting the variation in playing frequencies for clarinets. Proceedings of the Stockholm Music Acoustics Conference, 350-357, 2013.

[4] A. Hirschberg, J. Kergomard, and G. Weinreich. Mechanics of Musical Instruments, chapter 7, 291-361. Springer, New York, 1995.

[5] J. Van Zon, A. Hirschberg, J. Gilbert, and A. Wijnands. Flow through the reed channel of a single reed music instrument, J. Phys. Colloques, 51:C2-821-C2-824, 1990.

[6] P.A. Taillard, J. Kergomard, and F. Laloë. Iterated maps for clarinet-like systems. Nonlinear Dynamics, 62:253-271, 2010. 
[7] P.A. Taillard, J. Kergomard. An analytical prediction of the bifurcation scheme of a clarinetlike instrument: Effects of resonator losses. Acta Acustica united with Acustica101:2015, 279-291.

[8] J.P. Dalmont, P. Guillemain, and P.A. Taillard. Influence of the reed flow on the intonation of the clarinet. 1173-1177, Acoustics 2012 Nantes, 2012.

[9] M. van Walstijn, F. Avanzini, Modelling the mechanical response of the reed-mouthpiecelip system of a clarinet. part II: A lumped model approximation. Acta Acustica united with Acustica, 93:2007, 435-446.

[10] C.A. Macaluso, J.-P. Dalmont, Trumpet with near-perfect harmonicity: Design and acoustic results, J. Acoust. Soc. Am., 129: 404-414, 2011.

[11] P. Guillemain, J. Kergomard, and T. Voinier. Real-time synthesis of clarinet-like instruments using digital impedance models. J. Acoust. Soc. Am., 118:483-494, 2005.

[12] X. Boutillon. Analytical investigation of the flattening effect - the reactive power balance rule. J. Acoust. Soc. Am., 90:724-763, 1991.

[13] N. Grand, J Gilbert, and F. Laloë. Oscillation threshold of woodwind instruments. Acustica, 83:137-151, 1997.

[14] This approximation supposes that $\omega_{n}$ is sufficiently far from the other natural frequencies, including the (negative) $-\omega_{n}$. This can be easily checked by expanding the impedance around $\omega_{n}$, at the first order.

[15] T. Wilson and G. Beavers. Operating modes of the clarinet. J. Acoust. Soc. Am., 56:653-658, 1974.

[16] F. Silva, J. Kergomard, and C. Vergez. Interaction of reed and acoustic resonator in clarinetlike systems. J. Acoust. Soc. Am., 124:3284-3295, 2008. 
[17] J. Kergomard and A. Chaigne. Acoustics of Musical Instruments, in French, 712 pages. Belin, Paris, 2008.

[18] J. Kergomard and J. Gilbert. Analysis of some aspects of the reed role for cylindrical wind instruments (in French). In Proceedings of the 5ème congrès Français d'acoustique, 294$297,2000$.

[19] A.H. Benade. Fundamentals of musical acoustics. 608 pages. Oxford University Press, Oxford, 1976.

[20] J. Kergomard, S. Ollivier, and J. Gilbert. Calculation of the spectrum of self-sustained oscillators using a variable truncation method: Application to cylindrical reed instruments. Acustica United with Acta Acustica, 86:685-703, 2000.

[21] J.P. Dalmont, B. Gazengel, J. Gilbert, and J. Kergomard. Aspects of tuning and clean intonation in reed instruments. Applied Acoustics, 46:19-60, 1995.

[22] C.K. Madsen, F.A. Edmonson, and C.H. Madsen. Modulated frequency discrimination in relationship to age and musical training. J. Acoust Soc. Am., 46:1468-1472, 1969.

[23] C.J. Nederveen, J.-P. Dalmont. Mode locking effects on the playing frequency for fork fingerings on the clarinet. J. Acoust Soc. Am., 131:689-697,2012.

[24] V. Debut, J. Kergomard, and F. Laloe. Analysis and optimisation of the tuning of the twelfths for a clarinet resonator. Applied Acoustics, 2003.

[25] J. Gilbert, L Ruiz, and S. Gougeon. Influence of the temperature on the tuning of a wind instrument, in French. Proceedings of the Congrès Français d'Acoustique, 599-602, 2006.

[26] M.O. Van Walstijn, J.S. Cullen, and D.M. Campbell. Modeling viscothermal wave propagation in wind instrument air columns. Proceedings of the Institute of Acoustics, 19:413-418, 1997. 
[27] D. Noreland. An experimental study of temperature variations inside a clarinet. 446-449, Proceedings of the Stockholm Musical Acoustics Conference, 2013.

[28] J. Coltman. Acoustical analysis of the boehm flute. J. Acoust. Soc. Am., 65:499-506, 1979.

[29] J. Coltman. Resonance and sounding frequencies of the flute. J. Acoust. Soc. Am., 40:99-107, 1966.

[30] L. Fuks. Predictions and measurements of exhaled air effecs in the pitch of wind instruments. volume 19, 373-378, Proceedings of the Institute of Acoustics, 1997.

[31] L. Fuks and J. Sundberg. Blowing pressures in bassoon, clarinet oboe and saxophone. Acustica United with Acta Acustica, 85:267-277, 1999.

[32] J. Chen, J. Smith, and J. Wolfe. Pitch bending and glissandi on the clarinet: Roles of the vocal tract and partial tone hole closure. J. Acoust. Soc. Am., 126(3):1511-1520, 2009.

[33] A. Almeida, D. George, J. Smith, and J. Wolfe. The clarinet: How blowing pressures, lip force, lip position and reed hardness affect pitch, sound level and spectrum. J. Acoust. Soc. Am., 134:2247-2255, 2013.

[34] M. Tervaniemi,V. Just, S. Koelsch, A. Widmann, E. Schröger. Pitch discrimination accuracy in musicians vs nonmusicians: an event-related potential and behavioral study. Experimental Brain Research, 161(1):1-10, 2005. 


\section{List of Figures}

Figure 1: The frequency difference $N_{c e n t s}$ between the 1st impedance peak frequency (resonance frequency) and the playing frequency for Note 1 (fingering for E) of the clarinet. Note 1 values: $\zeta=0.3, \eta_{3}=-0.0201, F_{1}=1243 \mathrm{~Hz}$ and $f_{1}=146 \mathrm{~Hz}$. Solid lines represent the analytical results, while dotted lines represent the numerical results. The grey thick line represents the inharmonicity effect, the grey thin line represents the reed flow effect, the light grey thin line represents the reed dynamics effect and finally, the black thick line is the total of all three of these effects.

Figure 2: The frequency difference $N_{\text {cents }}$ for Note 12 (fingering for Eb) of the clarinet. Note 12 values: $\zeta=0.3, \eta_{3}=-0.0036, F_{1}=2490 \mathrm{~Hz}, f_{1}=277 \mathrm{~Hz}$. The color / line scheme and the scale match that of Figure 1

Figure 3: The frequency difference $N_{\text {cents }}$ for Note 17 (fingering for Ab) of the clarinet. Note 17 values: $\zeta=0.3, \eta_{3}=-0.0142, F_{1}=3338 \mathrm{~Hz}, f_{1}=369 \mathrm{~Hz}$. The color / line scheme and the scale match that of Figure 1.

Figure 4: Analytical length corrections $(\Delta \ell)$ values, in $\mathrm{mm}$, as a function of note number, in the first register, at the threshold of oscillation. Black thin line: inharmonicity effect, Thin dashed line: reed dynamics, Dark grey solid line: temperature effect, Grey squares: reed flow effect and finally, the thick black solid line is the total of all four effects. Realize that the plot for the inharmonicity effect is at zero for the $\gamma=\gamma_{t h}$ (a value that changes depending on Note number, generally around $0.33)$.

Figure 5: Analytical length corrections $(\Delta \ell)$ values, in $\mathrm{mm}$, as a function of note number, in the first register. The color / line scheme matches that of Figure 4. The data are represented for $\gamma=0.65$.

Figure 6: Top line (black dotted) is the difference, in cents between the first resonance frequencies (of a particular $\mathrm{Bb}$ professional model clarinet) and the equal-tempered scale frequencies using an averaged temperature of 26.5 degrees Celsius. The lower two lines represent the total frequency shift vs. the equal-tempered scale, in cents, as a function of note number, for the first register, 
${ }_{458}$ where $\gamma=0.65$ (black solid line) and $\gamma_{t h}$ (grey dotted line). 